\title{
How patient specific are patient-specific computational models of cerebral aneurysms? An overview of sources of error and variability
}

\author{
David A. Steinman, PhD, ${ }^{1}$ and Vitor M. Pereira, MD² \\ 1Department of Mechanical and Industrial Engineering and Institute of Biomaterials and Biomedical Engineering, University of \\ Toronto; and 2Divisions of Neuroradiology and Neurosurgery, Toronto Western Hospital, University Health Network, Toronto, \\ Ontario, Canada
}

Computational modeling of cerebral aneurysms, derived from clinical 3D angiography, has become widespread over the past 15 years. While such "image-based" or "patient-specific" models have shown promise for the assessment of rupture risk, much debate remains about their reliability in light of necessary modeling assumptions and incomplete or uncertain model input parameters derived from the clinic. The aims of this review were to walk through the various steps of this socalled patient-specific modeling pipeline and to highlight evidence supporting those steps that we can or cannot rely on. The relative importance of the different sources of error and variability on hemodynamic predictions is summarized, with recommendations to standardize for those that can be avoided and to pay closer attention those to that cannot.

https://thejns.org/doi/abs/10.3171/2019.4.FOCUS19123

KEYWORDS computational fluid dynamics; hemodynamics; wall shear stress; wall stress; image-based modeling

A $\mathrm{s}$ reviewed elsewhere in this issue, there is strong support for the idea that biomechanical forcesnotably wall shear stress (WSS) at the blood-wall interface and structural stresses within the wall-play a central role in cerebral aneurysm pathophysiology. As these forces are extremely difficult to measure clinically, there has been much interest in translating computational modeling, widely used in engineering practice, to the clinical domain.

Computational modeling of cerebral aneurysms derived from 3D angiography has been around since the early 2000s. ${ }^{54}$ Such modeling is referred to, often interchangeably, as "image-based" or "patient-specific," but the latter term is more stringent because, as discussed in this review, necessary input variables are not always available as part of routine patient care. Nevertheless, patient-specific (note: for the remainder of this review the quotation marks around "patient-specific" are implied) modeling has provided much of the evidence relating hemodynamic forces to aneurysm rupture, ${ }^{15}$ although the incremental value over traditional and morphological risk factors remains unclear..$^{50}$

Since the early 2010s, there has been growing clinical skepticism about the promise of patient-specific computational fluid dynamics (CFD). ${ }^{21,31,32}$ This, in turn, has triggered a more concerted effort on the part of engineers to understand, explain, and overcome possible pitfalls associated with modeling assumptions, incomplete clinical data, and inherent variabilities in the modeling pipeline. ${ }^{46,48,52,55,63}$

Per Fig. 1, computational modeling requires the 3D lumen (and, for structural modeling, the wall) and information about flow rates and/or pressures at the necessarily truncated boundaries (see Fig. 2). Also required are material properties of the blood and wall. Thus, how patientspecific a computational model is depends on how patient

ABBREVIATIONS CFD = computational fluid dynamics; ICA = internal carotid artery; MCA = middle cerebral artery; OSI = oscillatory shear index; TAWSS = time-averaged wall shear stress; $W S S=$ wall shear stress; $3 D R A=3 D$ rotational angiography.

SUBMITTED March 1, 2019. ACCEPTED April 12, 2019.

INCLUDE WHEN CITING DOI: 10.3171/2019.4.FOCUS19123. 


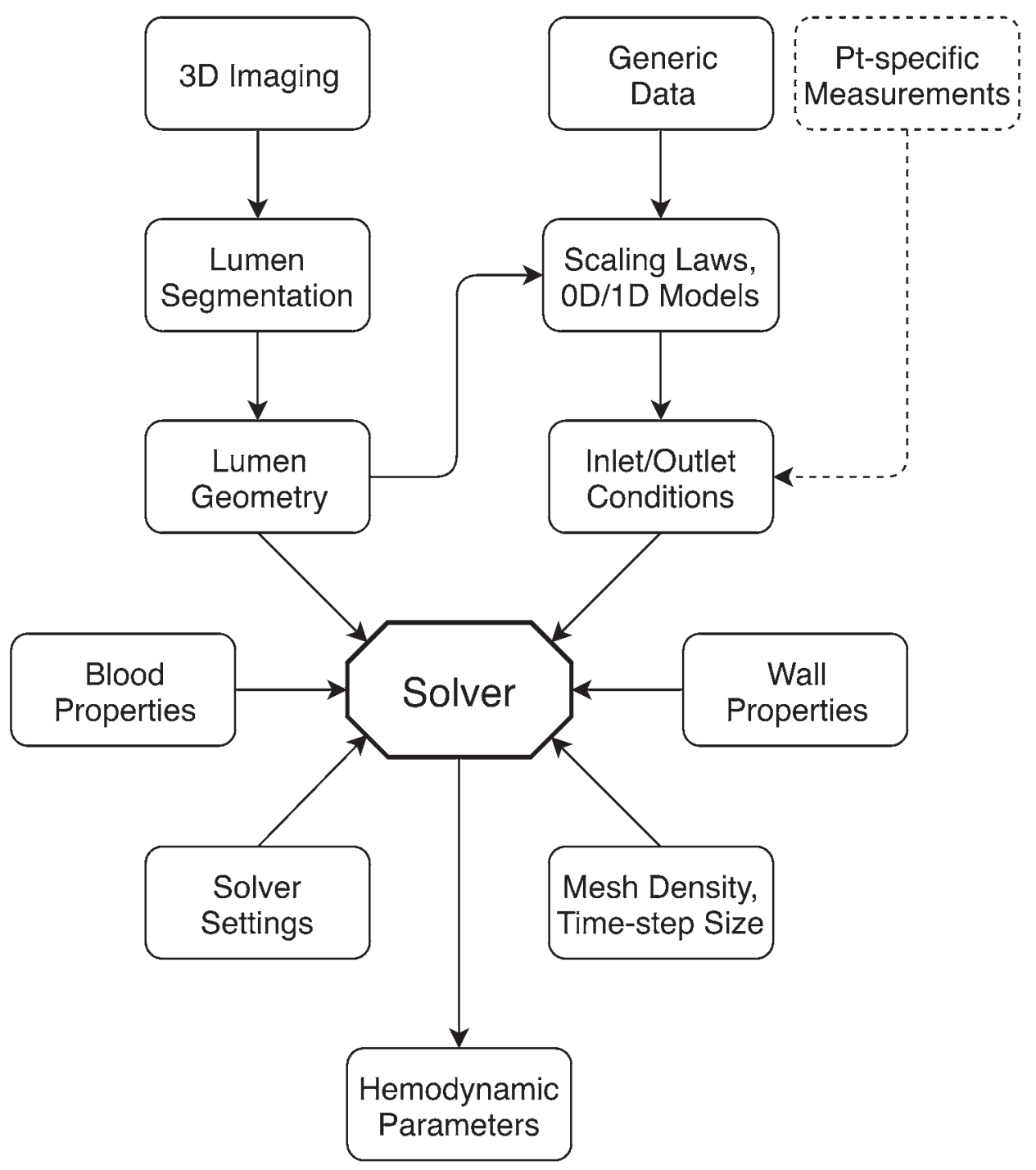

FIG. 1. Flowchart of typical steps/inputs into the patient-specific computational modeling pipeline. $\mathrm{Pt}=$ patient.

specific is this information. With that in mind, the aims of this review are as follows: 1) to explain the various and sometimes interacting sources of error and variability (note: "error" refers to the accuracy of the computational model relative to a [typically unknown] ground truth. "Variability" reflects the achievable precision due to interlaboratory variability in tools and techniques, intralaboratory variability in their use, and measurement or inherent physiological variability in input parameters); 2) to identify seminal studies that have investigated their impact on hemodynamic predictions; and 3) to discuss their relative importance in the context of how computational models might be used in clinical decision-making.

\section{Imaging and Segmentation}

\section{Imaging Modality}

Given that aneurysms have complicated, 3D morphologies, computational modeling must rely on 3D angiography. 3D rotational angiography (3DRA) provides the highest contrast and resolutions (approximately $0.2-0.3$ $\mathrm{mm}$ ) but is unsuitable for diagnosing and following patients with unruptured aneurysms. Modern multidetector CTA offers resolutions of approximately $0.4-0.5 \mathrm{~mm}$ but can still be challenged by small lesions, anatomical variations, and artifacts from nearby bone. MRA avoids ionizing radiation, but offers poorer resolutions (approximately $0.6-0.8 \mathrm{~mm}$ ) and may require contrast agents to overcome time-of-flight artifacts due to slowly recirculating flows that may be present in some aneurysms.

A 2011 CFD study of 10 aneurysms reconstructed from both 3DRA and CTA reported "excellent" agreement in gross flow patterns but "substantial" quantitative differences in time-averaged WSS (TAWSS); ${ }^{22}$ however, only a 4-slice CT scanner was used. A more recent comparison of 3DRA and cone-beam CTA for CFD of 4 aneurysms reported significant differences in low WSS ${ }^{37}$ nevertheless, qualitative differences in TAWSS appeared modest, at least relative to other sources of error/variability discussed in later sections. Our own preliminary CFD study of 16 

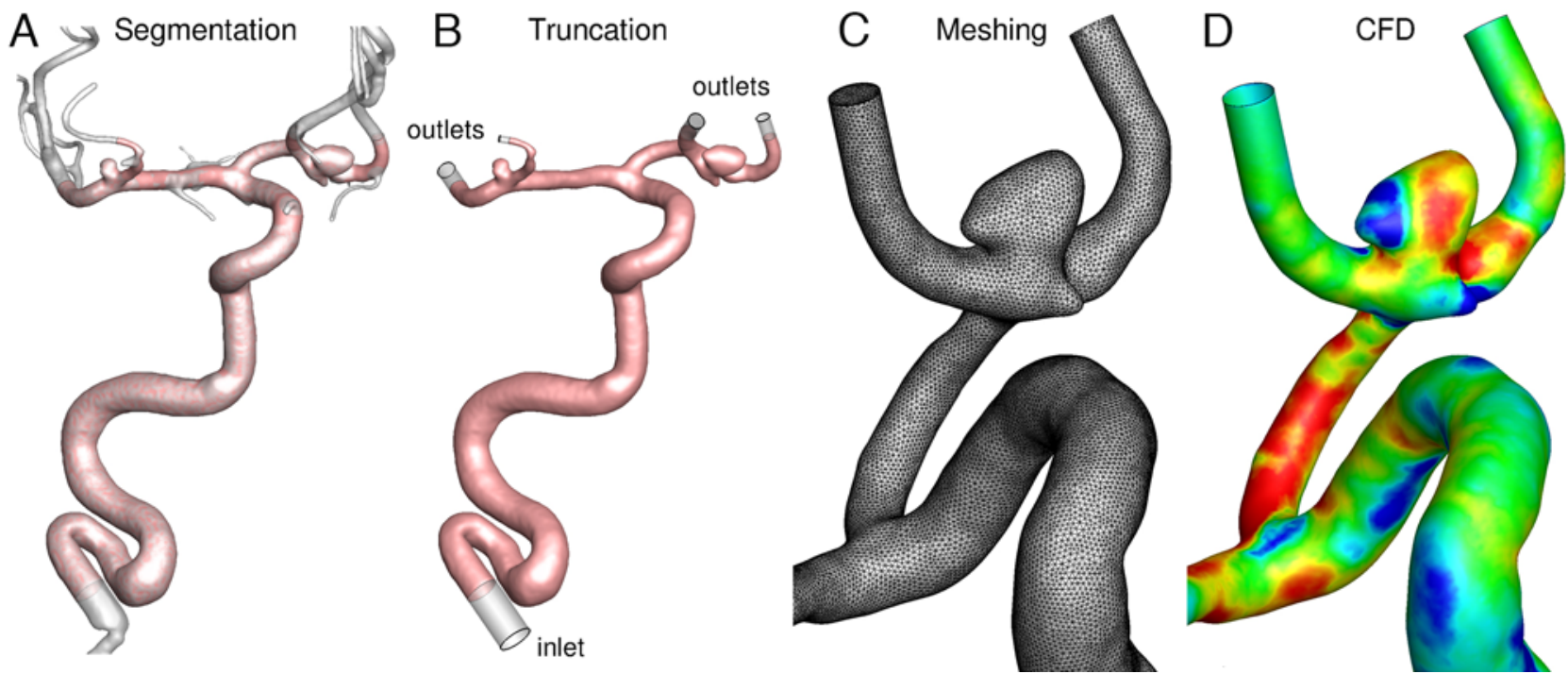

FIG. 2. Representative patient-specific CFD case derived from 3DRA, showing the stages from the following: lumen segmentation, smoothing, and removal of distal and small side branches (A); truncation and the addition of inlet and outlet flow extensions for imposing CFD boundary conditions (B); close-up view of the finite-element mesh, indirectly showing the density of volumetric tetrahedral elements from their visible triangular surfaces (C); and close-up view of the CFD result, showing TAWSS distribution (D).

aneurysms reconstructed using both 3DRA and modern (320-detector-row) 4DCTA suggested good agreement for TAWSS, at least for medium and large aneurysms.

With that said, Brinjikji et al. ' demonstrated that even 3DRA can overestimate the aneurysm neck compared to 2D DSA. Schneiders et al. ${ }^{49}$ subsequently reported neck overestimation in 8 of 20 3DRA-derived CFD models, which resulted in "non-negligible" errors in the size and/ or position of various hemodynamic features after manual correction of the neck from 2D DSA. Per Fig. 3, one can debate what constitutes "non-negligible"; however, it is indisputable that ostium area plays a central-but-complex role in intraaneurysmal flow dynamics: narrower ostia promote jetting and flow instability/turbulence but also resistance to flow entering the sac, suppressing jet momentum and instability.

In this regard, another source of variability is the (often site-specific or manufacturer-proprietary) filtering used to reconstruct image volumes from raw acquired data. ${ }^{44} \mathrm{~A}$ recent study of 8 aneurysms showed, for example, qualitative impact of 3DRA reconstruction kernel on peak systolic jetting, albeit with only modest changes in TAWSS. ${ }^{3}$

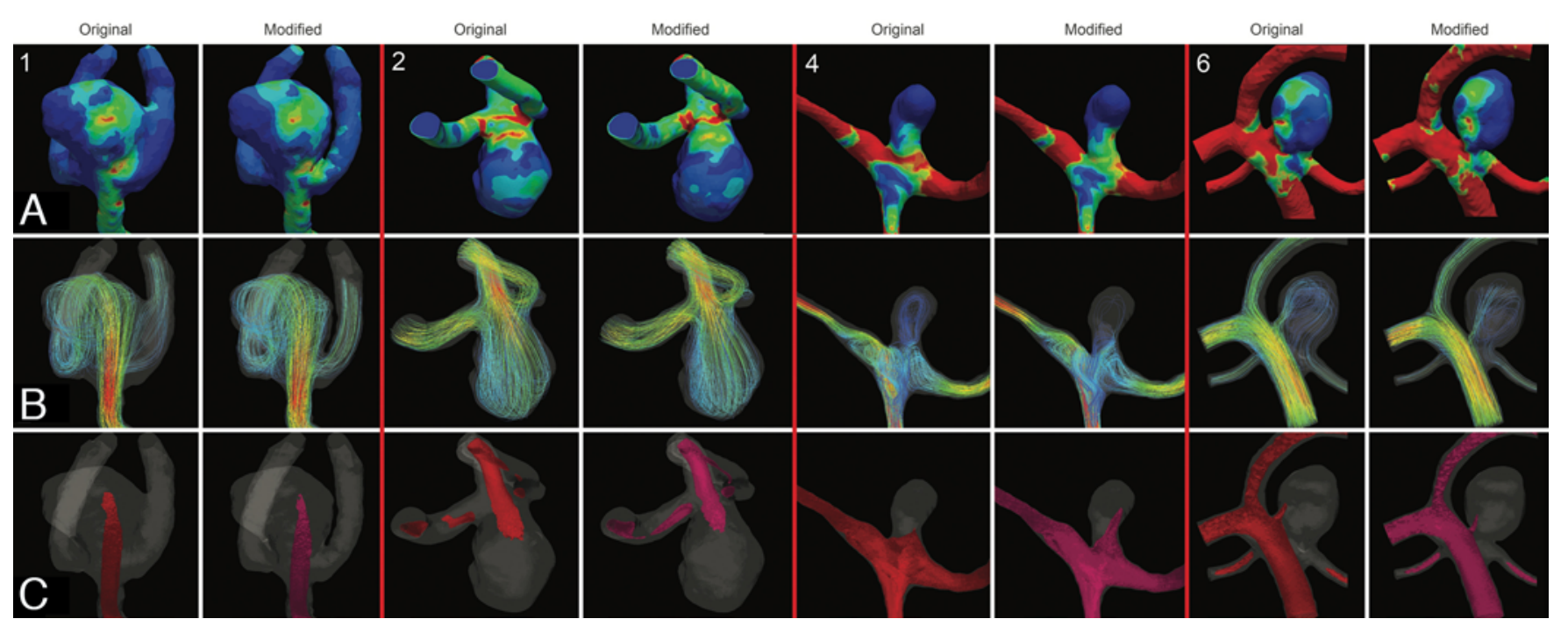

FIG. 3. Impact of neck segmentation errors for 4 representative aneurysm CFD cases reconstructed from 3DRA studies. Shown are TAWSS (A), streamlines (B), and velocity jetting before versus after neck modification based on 2D DSA (C). Republished with permission of the American Society of Neuroradiology. Intracranial aneurysm neck size overestimation with 3D rotational angiography: the impact on intra-aneurysmal hemodynamics simulated with computational fluid dynamics. Schneiders et al. ${ }^{49}$ AJNR Am J Neuroradiol 34:121-128, 2013. 


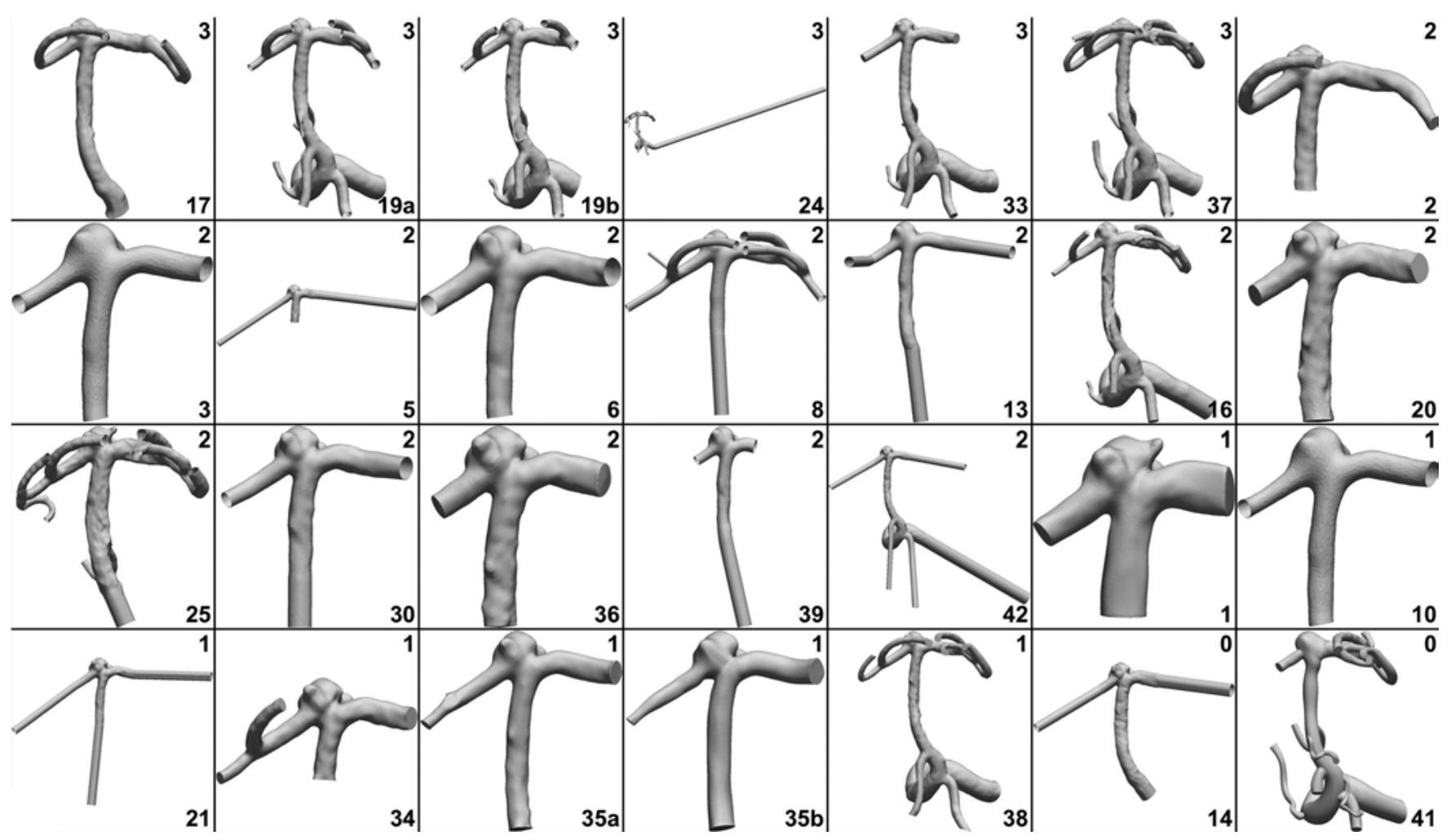

FIG. 4. Variability in lumen segmentation and model extent across 28 submissions to the 2015 Aneurysm CFD Challenge, for 1 of the $5 \mathrm{MCA}$ aneurysm cases. In each panel, the number at the top right is the self-reported expertise in aneurysm CFD, from 3 (highest) to 0 (lowest). The numbers at bottom right are the team ID numbers. From Valen-Sendstad et al. ${ }^{57}$ Image licensed under Creative Commons Attribution 4.0 International license CC BY 4.0 (https://creativecommons.org/licenses/by/4.0/). Used with permission.

Since hospital PACS may store only processed image volumes rather than raw data, the choice of reconstruction kernel can be irrevocable, exacerbating imaging variability and confounding standardization.

\section{Lumen Segmentation}

Segmentation to a specified image intensity (thresholding) is widely used for clinical visualization of 3D vasculature owing to its speed. For computational modeling, where modest variability in vascular diameter, roughness, or morphology can be amplified in hemodynamic parameters, segmentation to maximum intensity gradients is more attractive because, in theory anyway, they catch the true edge of the lumen regardless of any partial volume blurring. ${ }^{23}$

The "real world" variability of aneurysm segmentation for computational modeling purposes has been tested in two international CFD Challenges, for which participants were provided only with 3DRA image volumes. The first, in $2015,{ }^{57}$ revealed clear differences in sac morphology and roughness across the 26 participating groups. Per Fig. 4, it also highlighted another source of segmentation variability: some groups clipped their CFD models close to the sac, whereas others included more extensive distal and especially proximal vasculature. As discussed in the next section, this can have a nonnegligible impact on prescribed flow boundary conditions. While the design of the 2015 Challenge precluded isolating the impact of segmentation variability on hemodynamics, it is interesting to note that two groups each submitted two segmentations, differing in methodology, and/or smoothing. For one group there was only a $4 \%$ difference in TAWSS, but for the other it was up to $40 \%$, hinting that segmentation variability is a (the?) key contributor to variability in computational models.

The subsequent Multiple Aneurysms Anatomy Challenge (MATCH2018) ${ }^{4}$ found similar segmentation variability, despite roughly equal use of threshold- versus gradient-based methods among the 26 participating groups. Interestingly, only one group was deemed to have correctly segmented the aneurysm necks (vs blinded 2D DSA); however, they required nearly 24-person-hours/case compared to the median 2 hours. Unlike the design of the 2015 Challenge, MATCH2018 ultimately aimed to isolate the impact of segmentation variability on hemodynamic predictions versus other sources of input variability.

\section{Boundary Conditions}

In CFD, boundary conditions at inlets are usually imposed as time-varying velocities whose magnitudes are based on patient-specific or generic/estimated flow rates but whose cross-sectional profile shape must be assumed. At outlet(s), less restrictive conditions can be imposed that assume uniform pressure and/or negligible velocity gradients normal to each outlet's face. 


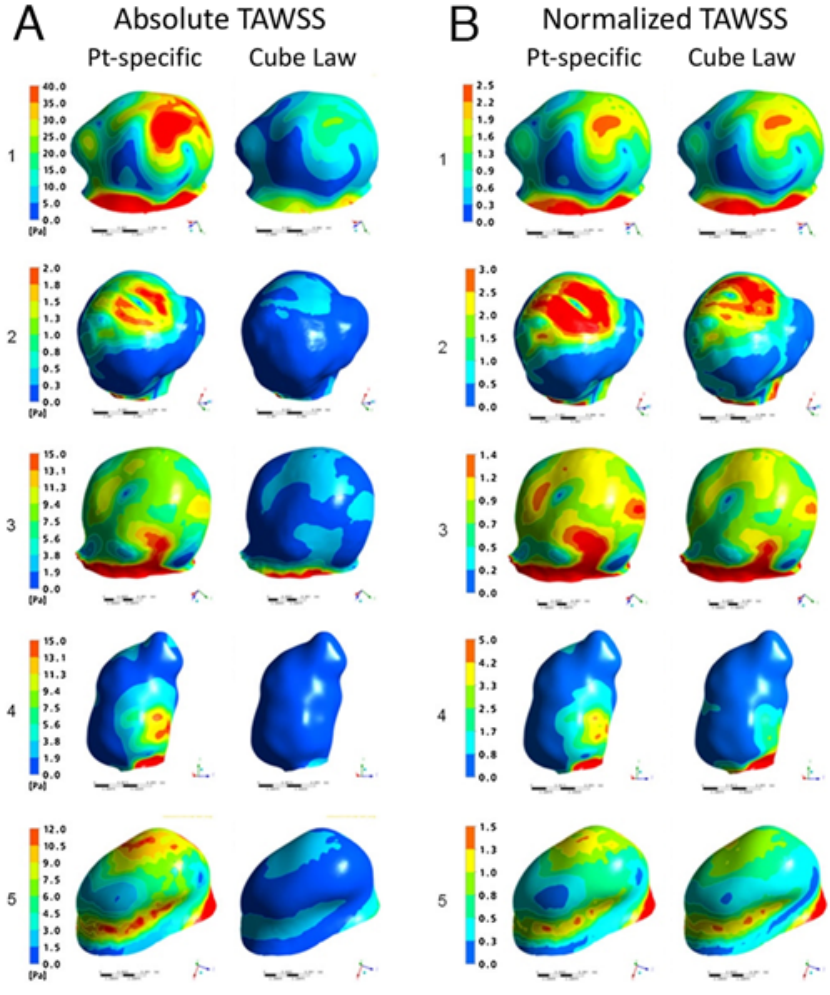

FIG. 5. Impact of flow rate boundary conditions on TAWSS distributions for 5 aneurysm CFD cases, based on patient-specific phase-contrast MRI measurements versus assuming a generic flow waveform with cycle-average flow rate proportional to the inlet diameter cubed. Panel B shows the same data as panel $\mathbf{A}$ but after normalizing each sac by its sac-average TAWSS in order to highlight similarities in TAWSS patterns despite the differences in TAWSS magnitudes. Reprinted with permission from Springer Nature, Ann Biomed Eng. Computational hemodynamics in cerebral aneurysms: the effects of modeled versus measured boundary conditions: Marzo et al. ${ }^{41}$ Copyright 2011.

\section{Inlet Velocity Profile Shape and Model Extent}

In the tortuous cerebrovasculature, the shapes of velocity profiles are complex and highly geometry dependent. So, for CFD, inlet velocity profiles are usually analytically prescribed to be uniform (i.e., flat or plug shaped) or fully developed (Poiseuille or Womersley), the latter equivalent to assuming a long cylindrical extension upstream. (Or, per Fig. 4, such long flow extensions may be used to "develop" a velocity profile computationally.)

As might be expected, the more patient specific the model is upstream of the aneurysm, the less dependent it will be on the assumed velocity profile shape., ${ }^{9,25,45}$ Yet, for the middle cerebral artery (MCA) aneurysm case in Fig. 4 , roughly half of the groups truncated the MCA to varying lengths, whereas others included the internal carotid artery (ICA) siphon. Coupled with the almost equal use of plug versus fully developed inlet velocity profiles, this was shown to contribute to the wide variability in the 2015 CFD Challenge. ${ }^{57}$

\section{Inflow Rates}

Patient-specific flow rates are rarely acquired as part of clinical routine. Instead, flow rates may be taken from measurements of another individual, averages from published cohorts, or exemplary network (0D/1D) models derived from them..$^{14,47}$ These, however, belie well-known variability in flow rates across patients.

A popular way around this is to assume a pulsatile flow waveform shape from the literature 27,28 and then scale its cycle-average (hereafter denoted $\mathrm{Q}_{\text {avg }}$ ) by the vessel diameter (D), reflecting that arteries tend to adapt their caliber to demanded flow. The most commonly used scaling law assumes $\mathrm{Q}_{\mathrm{avg}} \sim \mathrm{D}^{3}$, the so-called cube or Murray's law, ${ }^{42}$ which is tantamount to assuming the same inlet WSS for all patients. For large cerebral arteries, however, a square law $\left(\mathrm{Q}_{\mathrm{avg}} \sim \mathrm{D}^{2}\right)$, which assumes the same inlet mean velocity for all patients, has been shown to be more appropriate. ${ }^{11}$

That these scaling laws may be sources of error in aneurysm CFD has been demonstrated by several studies having access to patient-specific flow rates. Per Fig. 5, Marzo et $\mathrm{al}^{41}$ showed that a cube law tended to underestimate TAWSS, although its normalized distribution around the sac was surprisingly conserved. Conversely, Jansen et al. ${ }^{30}$ reported significant differences in TAWSS using patient-specific versus generic inflow conditions, highlighting qualitative differences in flow patterns for 21 of 36 cases. It is worth noting, however, that the authors actually imposed patient-specific $\mathrm{Q}_{\text {avg }}$ onto their generic flow waveforms, i.e., flow waveform shape was the only possible culprit. This is at odds with Xiang et al. ${ }^{61}$ who reported a negligible impact of waveform shape on TAWSS and oscillatory shear index (OSI) in 4 ICA aneurysms. Most recently, Chnafa et al. ${ }^{12}$ showed that use of any diameter scaling law can, for individual cases, introduce errors in $\mathrm{Q}_{\text {avg }}$ well above $50 \%$. In 3 of 4 cases, differences in TAWSS and OSI were negligible; however, in 1 case, differences even in TAWSS patterns were evident, and highfrequency flow instabilities appeared only when using the patient-specific flow rate.

In addition to introducing error, the use of different scaling laws, waveform shapes, and even diameter measurement location ${ }^{58}$ across modeling groups can introduce wide variability into the aneurysm CFD literature. This was demonstrated in the 2013 and 2015 CFD Challenges, which reported parent artery $\mathrm{Q}_{\text {avg }}$ varying by an order of magnitude across groups, ${ }^{29,57}$ and with some falling well outside normal physiological ranges.

\section{Outflow Rates or Pressures}

The most convenient way to handle multiple outlets in an aneurysm CFD model is to assume the same (often zero) pressure, which is the default setting in many CFD solvers, and is often justified-erroneously ${ }^{13}$-as the outlets feeding the same microvascular beds. Alternatively, the same scaling laws used for inlets can be used to estimate the division of flow across the outlets based on their diameters. While this has been shown to be reasonably accurate ${ }^{11}$ the flow divisions can depend on the (subjectively chosen) length and/or number of outlets. Moreover, for extensive models, the outflows actually determine the parent artery inflow rates (Fig. 2).

This was demonstrated by Chnafa et al., ${ }^{13}$ who proposed applying scaling laws not at each outlet but at each branch 


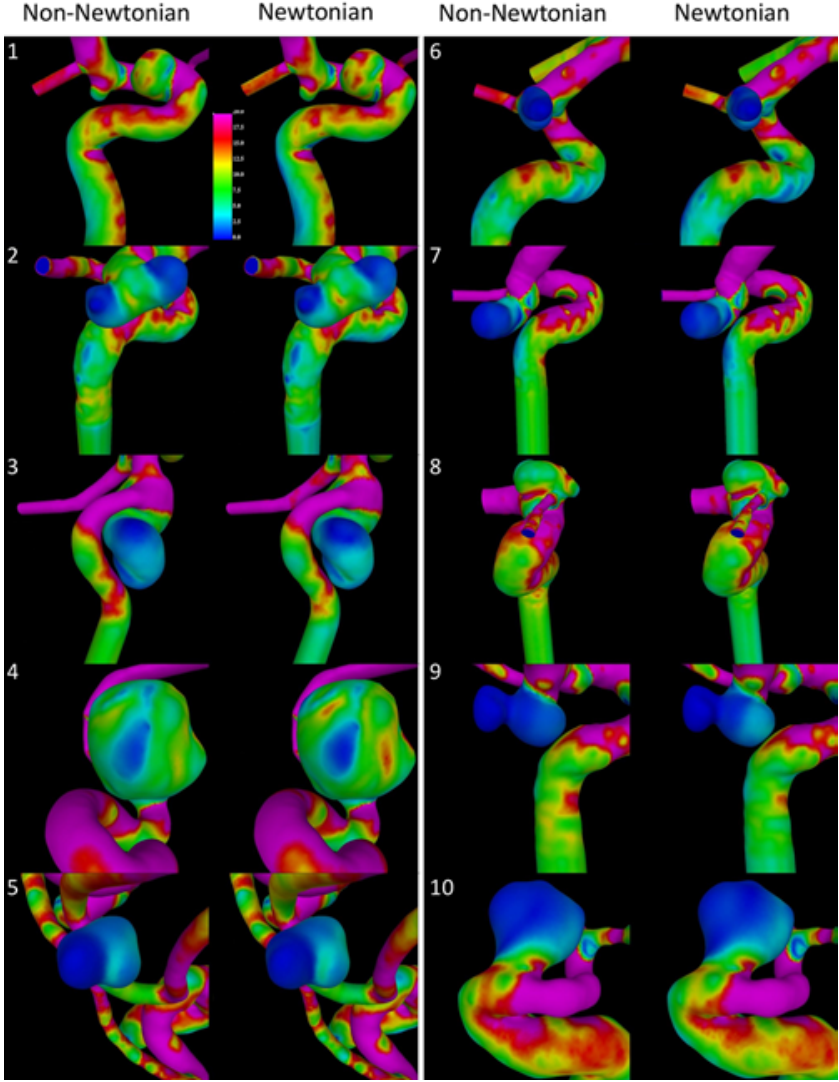

FIG. 6. Impact of non-Newtonian (shear-thinning) model on end-diastolic WSS for 10 aneurysm CFD cases. Reprinted with permission from Springer Nature. Med Biol Eng Comput: Unsteady wall shear stress analysis from image-based computational fluid dynamic aneurysm models under Newtonian and Casson rheological models. Castro et al. ${ }^{8}$ Copyright 2014.

point, essentially reducing the $3 \mathrm{D}$ model to a simple $0 \mathrm{D}$ network that predicted outflow rates or the outflow pressures required to achieve them. Based on 70 such $3 \mathrm{D} \rightarrow 0 \mathrm{D}$ MCA aneurysm cases, this "splitting" approach was shown to provide superior estimations of branch flow rates compared to the zero-pressure assumption, the latter also shown to be sensitive to arbitrary model truncations. In 10 cases selected for CFD, several showed differences in TAWSS and OSI between the splitting and zero-pressure approaches, which was attributable to differences in parent artery flow rates rather than aneurysm outflow divisions.

\section{Blood and Wall Properties Blood Properties}

Blood is a complex fluid having shear-thinning, viscoelastic, and thixotropic properties. Properly accounting for these non-Newtonian effects can be computationally prohibitive, so models typically assume constant (Newtonian) viscosity, considered reasonable given the nominally high shear rates and long time scales of flow in millimeter-sized arteries. When non-Newtonian models are employed computationally, they are almost invariably simple equations allowing viscosity to increase, instantaneously, with decreasing shear rate.
In 2012, Xiang et al. ${ }^{62}$ warned that a "Newtonian viscosity model could overestimate wall shear stress in intracranial aneurysm domes and underestimate rupture risk"; however, of the 3 cases studied, only 1, having a high aspect ratio and already pathologically low WSS, showed perceptible non-Newtonian effects. Conversely, Castro et al. ${ }^{8}$ concluded that non-Newtonian effects were not statistically significant, based on 10 cases showing only slight impact on WSS (Fig. 6). Similarly, Khan et al. ${ }^{34}$ concluded that non-Newtonian effects were negligible based on 3 cases for which CFD solver settings had demonstrably greater impact on hemodynamics.

So, while Newtonian viscosity might well overestimate WSS in regions of highly stagnant flow, like daughter sacs or blebs, ${ }^{24}$ it must be remembered that red blood cell aggregation (the mechanism for higher viscosity in stagnant flow) can require several cardiac cycles, whereas disaggregation may be instantaneous. ${ }^{16}$ Thus, simple shearthinning models may overestimate non-Newtonian effects as much as Newtonian models may underestimate them. ${ }^{1,38}$

Even for Newtonian models there is inherent error and variability. While most aneurysm patients obviously get blood work, viscosities, which can vary $\pm 20 \%$ among individuals,${ }^{6}$ are rarely provided to computational models. Even "normal" viscosity used by different groups can vary by approximately $10 \% .{ }^{57}$ Blood properties are thus almost never patient specific, but their impact on hemodynamic predictions is almost certainly secondary to geometry and flow rates, as discussed in previous sections.

\section{Wall Properties}

Most aneurysm CFD studies assume rigid walls, despite cerebral aneurysm walls tending to be highly heterogeneous, comprising thick, atherosclerotic or hypoplastic regions adjacent to superthin ones. These submillimeter thicknesses, let alone their intraindividual variations, are difficult to resolve with standard clinical imaging. Similarly, the material properties of these regions can also be highly variable. ${ }^{10}$ For this reason, most structural (or fluidstructure CFD) modeling of cerebral aneurysms has, historically, relied on assumed and often uniform wall properties, ${ }^{56}$ thus being hardly patient specific.

The potential impact of this was recently demonstrated by Voß et al., ${ }^{60}$ who reconstructed a patient-specific model of a fatal anterior communicating artery aneurysm imaged by micro-CT after autopsy and compared it to a model assuming constant thickness. As expected, wall stresses derived from the latter had substantial errors relative to the former. WSS, on the other hand, was already very low in this case and so the authors did not comment on any differences, except to note that time-averaged wall displacements were always below $1 \mathrm{~mm}$.

Indeed, it is well known from cine DSA that aneurysms tend to have small wall displacements. As opposed to trying to predict these based on gross assumptions about the wall, Dempere-Marco et al. ${ }^{17}$ directly imposed cine DSAderived displacements onto CFD models of 3 cases, revealing only slight differences in the magnitudes of WSS, and no overall change in their distributions, compared to rigid models. In a separate study by the same group, the 


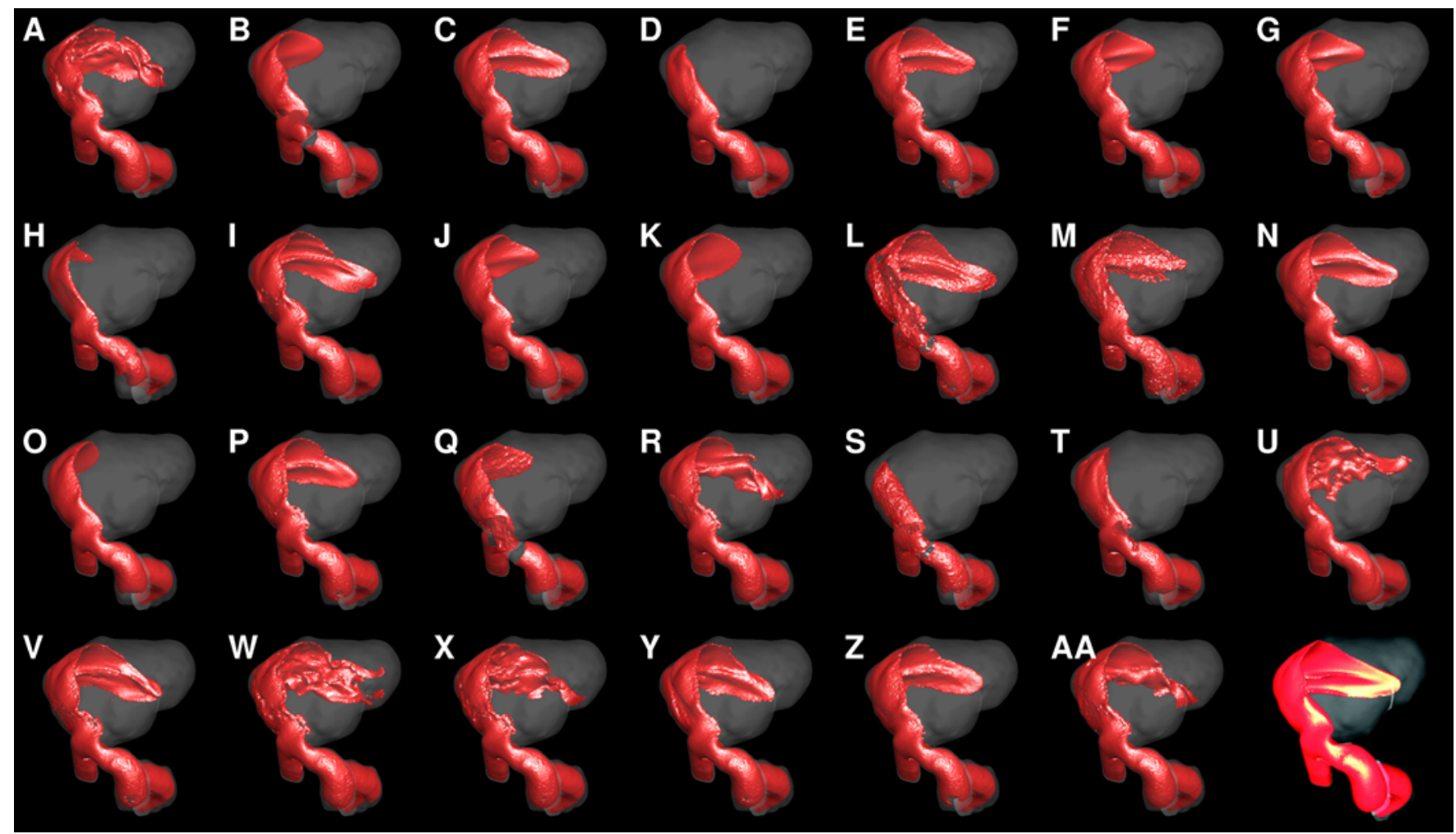

FIG. 7. Impact of solution strategy on peak systolic velocity jetting in a giant ICA aneurysm. Shown are the results from 27 submissions (labeled A-AA) compared to the original published result for this case, at lower right. From Steinman et al. ${ }^{53}$ Variability of computational fluid dynamics solutions for pressure and flow in a giant aneurysm: the ASME 2012 Summer Bioengineering Conference CFD Challenge. J Biomech Eng 135:021016, 2013. Republished with permission.

influence of parent artery motion on WSS in 2 basilar tip aneurysm cases was also shown to be negligible. ${ }^{51}$

Taken together, these observations support the almost universal assumption of rigid walls for patient-specific aneurysm CFD, thus avoiding the conceptual and computational complexities of fluid-structure modeling. They also help to explain the focus of cerebral aneurysm studies on WSS as a proxy for wall strength, in contrast to the successful application of wall stress in rupture risk prediction for aortic aneurysms ${ }^{20}$-namely, the larger size of the latter allows plausible resolution of the wall, without which any patient-specific predictions of wall stress would be meaningless.

\section{Solution Strategy}

In theory, if provided with all necessary patient-specific inputs, the output of a computational model should be error-free. In practice, of course, this is not the case because computational modeling relies on approximation of the governing equations by numerical schemes, discretization of the lumen and/or wall into a grid or mesh of points or elements, and division of the pulsatile cardiac cycle into discrete time steps. These choices are informed primarily by the trade-off between computational effort and accuracy.

To appreciate how this can introduce error and variability, consider the findings of the 2012 CFD Challenge, where all groups were provided with the same lumen ge- ometry, flow rates, and blood properties for a single ICA aneurysm case. ${ }^{53}$ Although gross quantities like pressure drops were largely consistent across groups, Fig. 7 shows that details like intraaneurysmal velocity jetting were highly variable, with only a handful of groups, using highresolution CFD, predicting flow instabilities.

This nonnegligible impact of solution strategy was subsequently demonstrated by Valen-Sendstad and Steinman, ${ }^{59}$ who showed, for 12 aneurysm cases, that typical numerical schemes, meshes, and time steps used in the aneurysm CFD literature could serve to suppress flow instabilities and alter WSS distributions compared to those predicted by higher-order schemes employing fine meshes and time-stepping. Khan et al. ${ }^{36}$ subsequently highlighted the dangers of relying on default numerical schemes and settings in commercial CFD solvers, whereas careful attention to these allowed for coarser meshes and time steps and ultimately faster simulations.

While there is growing awareness and reporting of numerical schemes and solver settings, $5,18,35$ mesh density is difficult to report owing to the use of different solver and element types and typically nonuniform refinement near walls and features of interest. Guidelines are thus difficult to provide, except to say that the onus is on individual groups to verify their solution strategies via proper mesh and time-step refinement studies. Nevertheless, in contrast to Hodis et al., ${ }^{26}$ who recommended case-by-case refinement, in clinical settings it would seem more practical to err on the side of caution with conservatively fine discreti- 
TABLE 1. Subjective ranking of relative importance of error/variability in patient-specific modeling pipeline and perceived prospects for standardization across modeling community

\begin{tabular}{|c|c|c|c|}
\hline Feature & $\begin{array}{l}\text { Impact of Error/ } \\
\text { Variability }\end{array}$ & $\begin{array}{l}\text { Prospects for } \\
\text { Standardization }\end{array}$ & Notes \\
\hline Imaging modality & Medium(?) & Low & $\begin{array}{l}\text { Difficult to standardize across sites but probably okay for many cases. } \\
\text { Impact of neck overestimation remains unclear. }\end{array}$ \\
\hline Segmentation & High(?) & Medium & $\begin{array}{l}\text { Gradient-based algorithms more objective than thresholding but subjec- } \\
\text { tive editing/smoothing a bigger source of variability. }\end{array}$ \\
\hline Domain extent & Medium & High & $\begin{array}{l}\text { Avoid short parent artery segments to minimize variability owing to inlet } \\
\text { velocity profile assumptions. }\end{array}$ \\
\hline Inflow rates & High & Medium & $\begin{array}{l}\text { Patient-specific data rare. Meta-analyses hampered by variety of diameter } \\
\text { scaling laws \& locations of diameter measurement. }\end{array}$ \\
\hline Outflow rates & Medium & High & $\begin{array}{l}\text { Scaling laws superior to widespread use of zero-pressure but still sensi- } \\
\text { tive to truncation or diameter measurement location. }\end{array}$ \\
\hline Blood properties & Low & High & $\begin{array}{l}\text { Non-Newtonian \&/or patient-specific viscosity not worth extra effort. Easy } \\
\text { to settle on standard values for viscosity, density. }\end{array}$ \\
\hline Wall properties & Low & High & $\begin{array}{l}\text { Structure or fluid-structure modeling highly sensitive to (unknown) wall } \\
\text { thickness \& properties. Rigid okay for CFD. }\end{array}$ \\
\hline Solver settings & Medium & Medium & $\begin{array}{l}\text { Commercial solver settings often opaque to users. Need gold-standard } \\
\text { computational data sets to properly tune settings. }\end{array}$ \\
\hline Mesh resolution & Medium & Medium & $\begin{array}{l}\text { Case-by-case refinement impractical. Aim for conservative recipes to } \\
\text { save person-hours at cost of (cheap) computation time. }\end{array}$ \\
\hline Time step size & Low & High & $\begin{array}{l}1000 \text { steps per cardiac cycle likely the minimum for capturing possible } \\
\text { high-frequency flow instabilities. }\end{array}$ \\
\hline Sac delineation & Low & Medium & $\begin{array}{l}\text { Minor impact on quantitation of hemodynamic parameters but need } \\
\text { consensus on handling branches arising out of sac. }\end{array}$ \\
\hline WSS parameters & Medium & High & $\begin{array}{l}\text { Need consensus on parameter definitions \& normalization to avoid same } \\
\text { terms meaning different things. }\end{array}$ \\
\hline
\end{tabular}

(?) indicates uncertainty as to medium or high.

zations, at the cost of (cheap) computation time but saving (expensive) operator time.

\section{Hemodynamic Parameters}

A great strength of computational modeling is its ability to derive any number of hemodynamic parameters. As pointed out by Kallmes, ${ }^{32}$ however, this is also one of its weaknesses: a veritable alphabet soup of proposed parameters, having various or vague definitions, and relying on ill-defined delineations of the sac and parent artery for averaging or normalization. For example, in a recent review, Liang et al. ${ }^{39}$ pointed out that low shear area, representing the fraction of the sac exposed to low WSS, relied on various criteria to define "low," ranging from a simple threshold value to some fraction of the "normal" parent artery WSS. Other quantities, like maximum WSS, could refer to the sac-averaged WSS at peak systole or the sac-maximum value of the time-averaged WSS, possibly contributing to its weak association with rupture.

As demonstrated by the 2015 CFD Challenge, some of the variability associated with segmentation and especially flow rate variability can be ameliorated by normalizing hemodynamic parameters to some patient-specific reference, e.g., parent artery WSS. Of course, this begs the question: how best to define the parent artery, especially for tortuous and tapering intracranial vessels? On the other hand, this same Challenge demonstrated that sac-average TAWSS reported by the groups was generally well correlated with sac-averaging performed by the organizers, suggesting that sac delineation may be a minor source of variability.

Finally, it is important to note that most of the abovecited studies have focused on TAWSS, owing to its nominal association with rupture. Other hemodynamic parameters related to the spatiotemporal dynamics of WSS can be expected to be more sensitive to errors or variability in inputs. $^{33}$

\section{Conclusions}

As detailed above, and summarized in Table 1, computational modeling of cerebral aneurysms is fraught with numerous sources of error and uncertainty, some unavoidable, some negligible, and some potentially reducible by establishing standards, protocols, and test cases ("phantoms"), echoing practice in clinical imaging. For example, truncation of models close to the aneurysm should be avoided to minimize the impact of the inevitable assumptions about velocity or pressure profile shape at boundary faces. Any perceived benefit of patient-specific blood properties is probably outweighed by the cost even of seeking them from medical records. Similarly, the substantial added cost of modeling nonrigid walls is difficult to justify in 
the face of substantial uncertainties in wall thickness and properties. While the cost-benefit of patient-specific flow rates is less clear, in their absence it would be helpful to reach some consensus on standard recipes for estimating inflow rates to facilitate meta-analyses, and to encourage reality checks of formulaically derived flow rates against physiological references..$^{40,64}$

In the end, most patient-specific computational models are patient specific in terms of their lumen geometry only, but still there may be errors introduced, especially around the critical neck, by even the best 3D imaging modalities. It is difficult to foresee standards for segmentation given the many subjective decisions required, so this may be a key application for machine learning.

Of course, these conclusions are themselves fraught with some uncertainty, in that most of the cited studies relied on a handful of cases, varying one input with all others fixed, and often-subjective judgments of what constituted non-negligible differences. In the future, studies using more robust uncertainty quantification methodologies, ${ }^{19}$ accounting for multiple interacting variabilities, are desired, as are studies testing the impact of these not on WSS itself but on its associations with more clinically oriented endpoints, e.g., wall pathology, enhancement, rupture, etc. Absent the latter, our review cannot presently draw conclusions about the fidelity of computational models for clinical decision-making.

Finally, we remind the reader that the alternative to computational modeling-direct clinical imaging of hemodynamics - is similarly fraught with oft-hidden assumptions and uncertainties. As just one example, spectral Doppler measurements of flow rates are of questionable reliability owing to highly skewed velocity profiles in the tortuous cerebrovasculature. ${ }^{43}$ So just as digital cameras rely on "computational photography," increasingly augmented by machine learning, to overcome innate hardware limitations, we may look forward to a time when computational modeling and medical imaging become more seamlessly integrated. ${ }^{2}$

\section{Acknowledgments}

This work was supported by Heart \& Stroke Foundation of Canada grant G-16-00012564.

\section{References}

1. Arzani A: Accounting for residence-time in blood rheology models: do we really need non-Newtonian blood flow modelling in large arteries? J R Soc Interface 15:20180486, 2018

2. Bakhshinejad A, Baghaie A, Vali A, Saloner D, Rayz VL, D'Souza RM: Merging computational fluid dynamics and 4D Flow MRI using proper orthogonal decomposition and ridge regression. J Biomech 58:162-173, 2017

3. Berg P, Saalfeld S, Voß S, Redel T, Preim B, Janiga G, et al: Does the DSA reconstruction kernel affect hemodynamic predictions in intracranial aneurysms? An analysis of geometry and blood flow variations. J Neurointerv Surg 10:290296, 2018

4. Berg P, Voß S, Saalfeld S, Janiga G, Bergersen AW, ValenSendstad K, et al: Multiple Aneurysms AnaTomy CHallenge 2018 (MATCH): Phase I: Segmentation. Cardiovasc Eng Technol 9:565-581, 2018

5. Botti L, Paliwal N, Conti P, Antiga L, Meng H: Modeling hemodynamics in intracranial aneurysms: Comparing accuracy of CFD solvers based on finite element and finite volume schemes. Int J Numer Methods Biomed Eng 34:e3111, 2018

6. Box FMA, van der Geest RJ, Rutten MCM, Reiber JHC: The influence of flow, vessel diameter, and non-Newtonian blood viscosity on the wall shear stress in a carotid bifurcation model for unsteady flow. Invest Radiol 40:277-294, 2005

7. Brinjikji W, Cloft H, Lanzino G, Kallmes DF: Comparison of 2D digital subtraction angiography and 3D rotational angiography in the evaluation of dome-to-neck ratio. AJNR Am J Neuroradiol 30:831-834, 2009

8. Castro MA, Ahumada Olivares MC, Putman CM, Cebral JR: Unsteady wall shear stress analysis from image-based computational fluid dynamic aneurysm models under Newtonian and Casson rheological models. Med Biol Eng Comput 52:827-839, 2014

9. Castro MA, Putman CM, Cebral JR: Computational fluid dynamics modeling of intracranial aneurysms: effects of parent artery segmentation on intra-aneurysmal hemodynamics. AJNR Am J Neuroradiol 27:1703-1709, 2006

10. Cebral JR, Duan X, Chung BJ, Putman C, Aziz K, Robertson AM: Wall mechanical properties and hemodynamics of unruptured intracranial aneurysms. AJNR Am J Neuroradiol 36:1695-1703, 2015

11. Chnafa C, Bouillot P, Brina O, Delattre BMA, Vargas MI, Lovblad KO, et al: Vessel calibre and flow splitting relationships at the internal carotid artery terminal bifurcation. Physiol Meas 38:2044-2057, 2017

12. Chnafa C, Bouillot P, Brina O, Najafi M, Delattre BMA, Vargas MI, et al: Errors in power-law estimations of inflow rates for intracranial aneurysm CFD. J Biomech 80:159-165, 2018

13. Chnafa C, Brina O, Pereira VM, Steinman DA: Better than nothing: a rational approach for minimizing the impact of outflow strategy on cerebrovascular simulations. AJNR Am J Neuroradiol 39:337-343, 2018

14. Chnafa C, Valen-Sendstad K, Brina O, Pereira VM, Steinman DA: Improved reduced-order modelling of cerebrovascular flow distribution by accounting for arterial bifurcation pressure drops. J Biomech 51:83-88, 2017

15. Chung B, Cebral JR: CFD for evaluation and treatment planning of aneurysms: review of proposed clinical uses and their challenges. Ann Biomed Eng 43:122-138, 2015

16. Cokelet GR: Rheology and hemodynamics. Annu Rev Physiol 42:311-324, 1980

17. Dempere-Marco L, Oubel E, Castro M, Putman C, Frangi A, Cebral J: CFD analysis incorporating the influence of wall motion: application to intracranial aneurysms. Med Image Comput Comput Assist Interv 9:438-445, 2006

18. Dennis KD, Kallmes DF, Dragomir-Daescu D: Cerebral aneurysm blood flow simulations are sensitive to basic solver settings. J Biomech 57:46-53, 2017

19. Eck VG, Donders WP, Sturdy J, Feinberg J, Delhaas T, Hellevik LR, et al: A guide to uncertainty quantification and sensitivity analysis for cardiovascular applications. Int J Numer Methods Biomed Eng 32:e02755, 2016

20. Fillinger MF, Marra SP, Raghavan ML, Kennedy FE: Prediction of rupture risk in abdominal aortic aneurysm during observation: wall stress versus diameter. J Vasc Surg 37:724-732, 2003

21. Fiorella D, Sadasivan C, Woo HH, Lieber B: Regarding "Aneurysm rupture following treatment with flow-diverting stents: computational hemodynamics analysis of treatment." AJNR Am J Neuroradiol 32:E95-E100, 2011 (Letter)

22. Geers AJ, Larrabide I, Radaelli AG, Bogunovic H, Kim M, Gratama van Andel HA, et al: Patient-specific computational hemodynamics of intracranial aneurysms from 3D rotational angiography and CT angiography: an in vivo reproducibility study. AJNR Am J Neuroradiol 32:581-586, 2011

23. Gremse F, Grouls C, Palmowski M, Lammers T, de Vries 
A, Grüll H, et al: Virtual elastic sphere processing enables reproducible quantification of vessel stenosis at CT and MR angiography. Radiology 260:709-717, 2011

24. Hippelheuser JE, Lauric A, Cohen AD, Malek AM: Realistic non-Newtonian viscosity modelling highlights hemodynamic differences between intracranial aneurysms with and without surface blebs. J Biomech 47:3695-3703, 2014

25. Hodis S, Kargar S, Kallmes DF, Dragomir-Daescu D: Artery length sensitivity in patient-specific cerebral aneurysm simulations. AJNR Am J Neuroradiol 36:737-743, 2015

26. Hodis S, Uthamaraj S, Smith AL, Dennis KD, Kallmes DF, Dragomir-Daescu D: Grid convergence errors in hemodynamic solution of patient-specific cerebral aneurysms. J Biomech 45:2907-2913, 2012

27. Hoi Y, Wasserman BA, Xie YJ, Najjar SS, Ferruci L, Lakatta EG, et al: Characterization of volumetric flow rate waveforms at the carotid bifurcations of older adults. Physiol Meas 31:291-302, 2010

28. Holdsworth DW, Norley CJ, Frayne R, Steinman DA, Rutt BK: Characterization of common carotid artery blood-flow waveforms in normal human subjects. Physiol Meas 20:219240, 1999

29. Janiga G, Berg P, Sugiyama S, Kono K, Steinman DA: The Computational Fluid Dynamics Rupture Challenge 2013Phase I: prediction of rupture status in intracranial aneurysms. AJNR Am J Neuroradiol 36:530-536, 2015

30. Jansen IG, Schneiders JJ, Potters WV, van Ooij P, van den Berg R, van Bavel E, et al: Generalized versus patient-specific inflow boundary conditions in computational fluid dynamics simulations of cerebral aneurysmal hemodynamics. AJNR Am J Neuroradiol 35:1543-1548, 2014

31. Kallmes DF: Identifying "truth" in computational fluid dynamics research. AJNR Am J Neuroradiol 32:E122-E123, 2011

32. Kallmes DF: Point: CFD_computational fluid dynamics or confounding factor dissemination. AJNR Am J Neuroradiol 33:395-396, 2012

33. Khan MO, Chnafa C, Gallo D, Molinari F, Morbiducci U, Steinman DA, et al: On the quantification and visualization of transient periodic instabilities in pulsatile flows. J Biomech 52:179-182, 2017

34. Khan MO, Steinman DA, Valen-Sendstad K: Non-Newtonian versus numerical rheology: Practical impact of shearthinning on the prediction of stable and unstable flows in intracranial aneurysms. Int J Numer Methods Biomed Eng 33:33, 2017

35. Khan MO, Valen-Sendstad K, Steinman DA: Cerebral aneurysm blood flow simulations: There's solver settings and then there's solver settings. J Biomech 61:280, 2017

36. Khan MO, Valen-Sendstad K, Steinman DA: Narrowing the expertise gap for predicting intracranial aneurysm hemodynamics: impact of solver numerics versus mesh and time-step resolution. AJNR Am J Neuroradiol 36:1310-1316, 2015

37. Lauric A, Hippelheuser JE, Malek AM: Critical role of angiographic acquisition modality and reconstruction on morphometric and haemodynamic analysis of intracranial aneurysms. J Neurointerv Surg 10:911-915, 2018

38. Lee SW, Steinman DA: On the relative importance of rheology for image-based CFD models of the carotid bifurcation. J Biomech Eng 129:273-278, 2007

39. Liang L, Steinman DA, Brina O, Chnafa C, Cancelliere NM, Pereira VM: Towards the clinical utility of CFD for assessment of intracranial aneurysm rupture-a systematic review and novel parameter-ranking tool. J Neurointerv Surg 11:153-158, 2019

40. MacDonald ME, Frayne R: Phase contrast MR imaging measurements of blood flow in healthy human cerebral vessel segments. Physiol Meas 36:1517-1527, 2015

41. Marzo A, Singh P, Larrabide I, Radaelli A, Coley S, Gwil- liam M, et al: Computational hemodynamics in cerebral aneurysms: the effects of modeled versus measured boundary conditions. Ann Biomed Eng 39:884-896, 2011

42. Murray CD: The physiological principle of minimum work: I. The vascular system and the cost of blood volume. Proc Natl Acad Sci U S A 12:207-214, 1926

43. Mynard JP, Steinman DA: Effect of velocity profile skewing on blood velocity and volume flow waveforms derived from maximum Doppler spectral velocity. Ultrasound Med Biol 39:870-881, 2013

44. O’Meara B, Rahal JP, Lauric A, Malek AM: Benefit of a sharp computed tomography angiography reconstruction kernel for improved characterization of intracranial aneurysms. Neurosurgery 10 (Suppl 1):97-105, 2014

45. Pereira VM, Brina O, Marcos Gonzales A, Narata AP, Bijlenga P, Schaller K, et al: Evaluation of the influence of inlet boundary conditions on computational fluid dynamics for intracranial aneurysms: a virtual experiment. J Biomech 46:1531-1539, 2013

46. Putman CM, Lylyk P, Cebral J: Reply regarding "Aneurysm rupture following treatment with flow-diverting stents: computational hemodynamics analysis of treatment." AJNR Am J Neuroradiol 32:E98-E100, 2011 (Letter)

47. Reymond P, Merenda F, Perren F, Rüfenacht D, Stergiopulos $\mathrm{N}$ : Validation of a one-dimensional model of the systemic arterial tree. Am J Physiol Heart Circ Physiol 297:H208H222, 2009

48. Robertson AM, Watton PN: Computational fluid dynamics in aneurysm research: critical reflections, future directions. AJNR Am J Neuroradiol 33:992-995, 2012

49. Schneiders JJ, Marquering HA, Antiga L, van den Berg R, VanBavel E, Majoie CB: Intracranial aneurysm neck size overestimation with 3D rotational angiography: the impact on intra-aneurysmal hemodynamics simulated with computational fluid dynamics. AJNR Am J Neuroradiol 34:121-128, 2013

50. Schneiders JJ, Marquering HA, van Ooij P, van den Berg R, Nederveen AJ, Verbaan D, et al: Additional value of intraaneurysmal hemodynamics in discriminating ruptured versus unruptured intracranial aneurysms. AJNR Am J Neuroradiol 36:1920-1926, 2015

51. Sforza DM, Löhner R, Putman C, Cebral J: Hemodynamic analysis of intracranial aneurysms with moving parent arteries: basilar tip aneurysms. Int J Numer Methods Biomed Eng 26:1219-1227, 2010

52. Steinman DA: Computational modeling and flow diverters: a teaching moment. AJNR Am J Neuroradiol 32:981-983, 2011

53. Steinman DA, Hoi Y, Fahy P, Morris L, Walsh MT, Aristokleous N, et al: Variability of computational fluid dynamics solutions for pressure and flow in a giant aneurysm: the ASME 2012 Summer Bioengineering Conference CFD Challenge. J Biomech Eng 135:021016, 2013

54. Steinman DA, Milner JS, Norley CJ, Lownie SP, Holdsworth DW: Image-based computational simulation of flow dynamics in a giant intracranial aneurysm. AJNR Am J Neuroradiol 24:559-566, 2003

55. Strother CM, Jiang J: Intracranial aneurysms, cancer, x-rays, and computational fluid dynamics. AJNR Am J Neuroradiol 33:991-992, 2012

56. Torii R, Oshima M, Kobayashi T, Takagi K, Tezduyar TE: Influencing factors in image-based fluid-structure interaction computation of cerebral aneurysms. Int J Numer Methods Fluids 65:324-340, 2011

57. Valen-Sendstad K, Bergersen AW, Shimogonya Y, Goubergrits L, Bruening J, Pallares J, et al: Real-world variability in the prediction of intracranial aneurysm wall shear stress: The 2015 International Aneurysm CFD Challenge. Cardiovasc Eng Technol 9:544-564, 2018

58. Valen-Sendstad K, Piccinelli M, KrishnankuttyRema R, 
Steinman DA: Estimation of inlet flow rates for image-based aneurysm CFD models: where and how to begin? Ann Biomed Eng 43:1422-1431, 2015

59. Valen-Sendstad K, Steinman DA: Mind the gap: impact of computational fluid dynamics solution strategy on prediction of intracranial aneurysm hemodynamics and rupture status indicators. AJNR Am J Neuroradiol 35:536-545, 2014

60. Voß S, Glaßer S, Hoffmann T, Beuing O, Weigand S, Jachau $\mathrm{K}$, et al: Fluid-structure simulations of a ruptured intracranial aneurysm: constant versus patient-specific wall thickness. Comput Math Methods Med 2016:9854539, 2016

61. Xiang J, Siddiqui AH, Meng H: The effect of inlet waveforms on computational hemodynamics of patient-specific intracranial aneurysms. J Biomech 47:3882-3890, 2014

62. Xiang J, Tremmel M, Kolega J, Levy EI, Natarajan SK, Meng $\mathrm{H}$ : Newtonian viscosity model could overestimate wall shear stress in intracranial aneurysm domes and underestimate rupture risk. J Neurointerv Surg 4:351-357, 2012

63. Xiang J, Tutino VM, Snyder KV, Meng H: CFD: computational fluid dynamics or confounding factor dissemination? The role of hemodynamics in intracranial aneurysm rupture risk assessment. AJNR Am J Neuroradiol 35:1849-1857, 2014
64. Zarrinkoob L, Ambarki K, Wåhlin A, Birgander R, Eklund A, Malm J: Blood flow distribution in cerebral arteries. J Cereb Blood Flow Metab 35:648-654, 2015

\section{Disclosures}

The authors report no conflict of interest concerning the materials or methods used in this study or the findings specified in this paper.

\section{Author Contributions}

Conception and design: Steinman. Analysis and interpretation of data: Steinman. Drafting the article: Steinman. Critically revising the article: both authors. Reviewed submitted version of manuscript: both authors. Approved the final version of the manuscript on behalf of both authors: Steinman.

\section{Correspondence}

David A. Steinman: University of Toronto, ON, Canada. steinman@mie.utoronto.ca. 